\title{
Combination of albumin concentration and neutrophil-to- lymphocyte ratio for predicting overall survival of patients with non-small cell lung cancer
}

\author{
Jinsen Weng ${ }^{1 \#}$, Jieping Huang ${ }^{2 \#}$, Wei Yu ${ }^{3 \#}$, Zhiyong Zhao ${ }^{4}$, Biao Zhu ${ }^{4}$, Jingping Lin ${ }^{1}$, Yibin Cai ${ }^{5}$, \\ Jiulong Zhang, Weikun Su${ }^{5}$, Xiaohui Chen ${ }^{5}$, Kunshou Zhu ${ }^{5}$, Shaofeng Lin ${ }^{5}$ \\ ${ }^{1}$ Department of Critical Care Medicine, Fujian Medical University Cancer Hospital \& Fujian Cancer Hospital, Fuzhou, China; ${ }^{2}$ Emergency \\ Department of Fujian Medical University Union Hospital, Fuzhou, China; ${ }^{3}$ Department of Clinical Pharmacy, Fujian Medical University Cancer \\ Hospital \& Fujian Cancer Hospital, Fuzhou, China; ${ }^{4}$ Department of Critical Care Medicine, Fudan University Shanghai Cancer Center, Shanghai, \\ China; ${ }^{5}$ Department of Thoracic Surgery, Fujian Medical University Cancer Hospital \& Fujian Cancer Hospital, Fuzhou, China \\ Contributions: (I) Conception and design: K Zhu, S Lin; (II) Administrative support: Z Zhao, B Zhu; (III) Provision of study materials or patients: \\ J Lin, Y Cai, J Zhang; (IV) Collection and assembly of data: W Su, X Chen; (V) Data analysis and interpretation: J Weng, J Huang, W Yu; (VI) \\ Manuscript writing: All authors; (VII) Final approval of manuscript: All authors. \\ "These authors contributed equally to this work. \\ Correspondence to: Shaofeng Lin; Kunshou Zhu. Department of Thoracic Surgery, Fujian Medical University Cancer Hospital \& Fujian Cancer \\ Hospital, Fuzhou 350014, China. Email: linsf@fjzlhospital.com; zhuks@fjzlhospital.com.
}

Background: Lung cancer contributes significantly to the total of cancer-linked deaths globally, accounting for 1.3 million deaths each year. Preoperative albumin (Alb) concentration and neutrophil-to-lymphocyte ratio (NLR) may reflect chronic inflammation and be used to predict lung cancer outcomes.

Methods: The clinical records of 293 patients with non-small cell lung cancer (NSCLC) in Fujian Medical University Cancer Hospital \& Fujian Cancer Hospital were reviewed retrospectively in this current study. Clinicopathologic pretreatment, including NLR, Glasgow prognostic score (GPS), and post-treatment value, such as tumor-node-metastasis (TNM) were documented. The cut-off finder application was employed to calculate the optimal threshold values. The significance of Alb concentration combined with NLR (COANLR) on the prediction of overall survival (OS) was explored using Kaplan-Meier analysis along with Cox proportional hazards.

Results: The results revealed that COA-NLR could independently assess the OS of patients with NSCLC [hazard ratio $(\mathrm{HR})=1.952,95 \%$ confidence interval (CI): 1.367 to 2.647, $\mathrm{P}<0.001$ ]. Moreover, the 3 -year OS rates were $87.2 \%, 68.5 \%$, and $52.8 \%$ for the COA-NLR $=0, \mathrm{COA}-\mathrm{NLR}=1$, and COA-NLR $=2$, respectively $(\mathrm{P}<0.001)$.

Conclusions: Preoperative COA-NLR value can effectively stratifies prognosis in NSCLC patients by classified patients into three independent groups. It can be adopted as an effective biomarker for prognosis in NSCLC patients treated with resection.

Keywords: Albumin (Alb); prognosis; neutrophil-to-lymphocyte ratio (NLR); non-small cell lung cancer (NSCLC)

Submitted Jul 02, 2021. Accepted for publication Sep 02, 2021.

doi: $10.21037 /$ jtd-21-1320

View this article at: https://dx.doi.org/10.21037/jtd-21-1320

\section{Introduction}

Lung cancer contributes significantly to the total number of cancer-linked deaths globally, is responsible for
1.3 million deaths each year (1), and the most common cancer diagnosed in men in China. Among lung cancers, non-small-cell lung cancer (NSCLC) constitutes $85 \%$ 
of all primary lung cancers (2). Although remarkable advancements have been made in the treatment as well as clinical diagnosis of NSCLC, the median survival of this cancer remains unsatisfactory.

Predicting the outcomes in individual patients is difficult. Previously, the tumor-node-metastasis (TNM) system, genetic biomarkers, along with histological subtype served as the common predictive tools for estimating overall survival (OS) (3). Nevertheless, patients in the TNM stage or with the histopathological subtype exhibited remarkably diverse survival, and due to the high cost of genetic biomarkers, their clinical use was limited.

The systemic inflammatory response (SIR) plays an important role in the lung cancer prognosis. The biomarkers include C-reactive protein (CRP), albumin (Alb), neutrophilto-lymphocyte ratio (NLR) and fibrinogen (Fib), which have been commonly observed in lung cancer patients. Of these, low Alb concentration are associated with poor survival, and preoperative serum Alb concentration can accurately predicted long-term outcomes in patients with cancer. Meanwhile, NLR has been candidate signatures to reveal the status of chronic inflammation and estimate the outcome in lung cancer patients (4-6) Therefore, we hypothesized that the combination of Alb and NLR would be better predict the prognosis in NSCLC. In this paper, we developed a new inflammation-based prognostic system, referred to as the combination of Alb concentration and NLR (COA-NLR), which may better reflect systemic inflammation level and stratify the patients into different risk categories.

Herein, we verified the prognostic significance of Alb and NLR in individuals with NSCLC treated with lung resection. Moreover, we also evaluated the relationship of COA-NLR groups with the clinic-pathologic features or the clinical laboratory variables.

We present the following article in accordance with the REMARK reporting checklist (available at https://dx.doi. org/10.21037/jtd-21-1320).

\section{Methods}

\section{Eligible patients}

This retrospective study examined data of NSCLC patients who had undergone pulmonary resection (pneumonectomy or lobectomy) and dissection of the hilar, as well as mediastinal lymph nodes at the Fujian Provincial Tumor Hospital, China, between January 2006 and January 2017. All NSCLC patients were newly diagnosed, then classified via clinical symptoms along with pathological grading, per the international association guidelines for lung cancer TNM staging ( th $^{\text {th }}$ edition of the TNM classification). All participants had undergone surgical resection, and some had been given adjuvant radiotherapy or chemotherapy when needed. Patients with hematological disease, autoimmune disease, and infection were excluded. On the basis of the inclusion and exclusion criteria above, a total of 293 NSCLC patients were analyzed. The study was conducted in accordance with the Declaration of Helsinki (as revised in 2013). This study was approved by the Ethics Committee of Fujian Medical University Cancer Hospital \& Fujian Cancer Hospital, China, and the need for informed consent was waived given the use of retrospective data.

We collected clinic-pathologic findings of the 293 participants, including age, Glasgow prognostic score (GPS), TNM stage, and blood cell count. Within 1 week before surgery, we collected peripheral venous blood from each participant between $8 \mathrm{am}$ and $10 \mathrm{am}$. The $7^{\text {th }}$ edition of the TNM classification was employed to assign tumor stages (7).

\section{Definition of COA-NLR}

Through dividing the neutrophil numbers by the absolute lymphocyte numbers, the NLR was determined. The receiver operating characteristic (ROC) curves were adopted to explore the threshold values of the pre-operative NLR, as well as Alb. The recommended NLR threshold value was determined on the basis of the predominant point on the ROC curve for sensitivity (0.429) and specificity (0.700), respectively. The two parameters yielded a threshold value of 2.565; therefore, we defined the recommended threshold value of NLR as 2.5. The area under the ROC curve (AUC) was 0.672 . Besides, the recommended preoperative Alb threshold value was defined as $35 \mathrm{~g} / \mathrm{L}$, since the dominant point on the ROC curve yielded a threshold value of $35.7 \mathrm{~g} / \mathrm{L}$ for sensitivity (0.514), as well as specificity $(0.785)$. The AUC was 0.644 .

The COA-NLR was computed based on data obtained on the day of admission as shown: patients exhibiting an elevated NLR $(>2.5)$ and reduced Alb value $(<35 \mathrm{~g} / \mathrm{L})$ were designated a score of 2 , and patients exhibiting either, or neither, were designated a score of 1 or 0 , respectively.

\section{Follow up}

A regular follow-up examination was conducted once every 
6 months to determine the survival data via retrieval of medical records, email, as well as telephone, with 31 January 2017 being the deadline of the follow-up. We defined OS time as the time from the first treatment to death or last follow-up.

\section{Statistical analysis}

All recorded data are presented as mean \pm standard deviation (SD). The chi-squared test along with the Kruskal-Wallis test were adopted to determine the differences among groups.

To determine the optimal cutoff values of each clinical laboratory variable, a ROC curve was created, and the cutoff values were set as the point on the curve that was closest to the upper left-hand corner of the plot.

The OS rates were determined using the Kaplan-Meier approach, and comparisons were determined by log-rank test. Each variable was assessed on the univariate analysis, and then calculated on the multivariable Cox proportional hazards model to validate their independent prognostic value. Statistical analyses were performed using the software SPSS version 22.0 (SPSS Inc., Chicago, IL, USA). A P value $<0.05$ indicated statistical significance.

\section{Results}

\section{Patients characteristics}

Overall, 293 consecutive patients with complete medical data and follow-up data were enrolled. The distribution of the clinical background features of the three groups of participants were divided according to the COA-NLR, as shown in Table 1. The results did not reveal any remarkable differences among the groups, except for gender, tobacco, GPS, tumor status, and TNM status.

\section{Prognostic analysis}

Table 2 demonstrates that OS, CRP, white blood cell (WBC), neutrophil, lymphocyte, NLR, hemoglobin $(\mathrm{Hb})$, platelet (PLT), Alb, glucose, and lactate dehydrogenase (LDH) were significantly associated with the three groups of NSCLC patients.

During the observation period, 70 (23.9\%) participants died. Univariate analysis along with multivariate Cox proportional hazards model assessments were performed to explore the relationship between clinical characteristics and OS.

Univariate analysis revealed that adjuvant therapy $(\mathrm{P}=0.003)$, COA-NLR $(\mathrm{P}<0.001)$, GPS $(\mathrm{P}<0.001)$, WBC $(\mathrm{P}<0.001)$, neutrophil $(\mathrm{P}<0.001)$, NLR $(\mathrm{P}<0.001)$, PLT $(\mathrm{P}=0.001)$, Alb $(\mathrm{P}<0.001)$, alanine transaminase $(\mathrm{ALT})$ $(\mathrm{P}=0.031)$, glucose $(\mathrm{P}=0.019)$, lymph status $(\mathrm{P}<0.001)$, tumor status $(\mathrm{P}=0.001)$, metastasis $(\mathrm{P}=0.004)$ and $\mathrm{TNM}$ status $(\mathrm{P}<0.001)$ were significantly associated with $\mathrm{OS}$ in patients with NSCLC. Age, gender, body mass index (BMI), smoking, CRP, Hb, and LDH did not estimate OS following the surgery (Table 3).

Multivariate assessments showed that COA-NLR [hazard ratio $(\mathrm{HR})=1.952,95 \%$ confidence interval $(\mathrm{CI}): 1.367$ to $2.647, \mathrm{P}<0.001$ ], lymph ( $\mathrm{HR}=0.486,95 \% \mathrm{CI}: 0.260$ to 0.985, $\mathrm{P}=0.045$ ), PLT (HR $=1.878,95 \%$ CI: 1.403 to 3.970 , $\mathrm{P}=0.007)$, glucose $(\mathrm{HR}=1.450,95 \% \mathrm{CI}: 1.125$ to 3.443 , $\mathrm{P}=0.018$ ), and TNM status (HR $=1.852,95 \% \mathrm{CI}: 1.523$ to $2.547, \mathrm{P}<0.001$ ) were independent predictive factors (Table 4).

The Kaplan-Meier approach was employed to create OS curves, as stratified on the basis of the COA-NLR score and TNM status. The 3 -year OS rates were $87.2 \%, 68.5 \%$, and $52.8 \%$ for the COA-NLR $=0$, COA-NLR $=1$, and COA$\mathrm{NLR}=2$, respectively. The log-rank test was used to explore the differences in survival, which exhibited remarkable distinctions for all strata $(\mathrm{P}<0.001$, Figure 1).

\section{Discussion}

Herein, we found that patients with COA-NLR 1 or 2 have lower GPS, higher T stage, and higher TNM stage in contrast with those with COA-NLR 0 (Table 1). Similarly, NSCLC patients with lower GPS, higher T stage, and higher TNM stage had poor OS (Table 3). As COA-NLR constitutes systemic inflammatory response syndrome (SIRS)-linked characteristics-NLR and Alb level, it may well reflect the severity of the disease, so it was reasonable to find significant differences between COA-NLR groups regarding GPS, T stage, and TNM stage. Therefore, as predicted, we found that there were also significant differences between OS of NSCLC patients and traditional severity indexes, such as GPS, T stage, and TNM stage in NSCLC patients.

Furthermore, we also established that COA-NLR was an independent predictive factor, COA-NLR with a lower score was linked to better OS in individuals with NSCLC. The NLR is an index of circulating immune cells; the new predictor, COA-NLR, may better reveal the balance of systemic inflammation with host immune response for the 
Table 1 Relationships of clinical characteristics with the COA-NLR in individuals with NSCLC

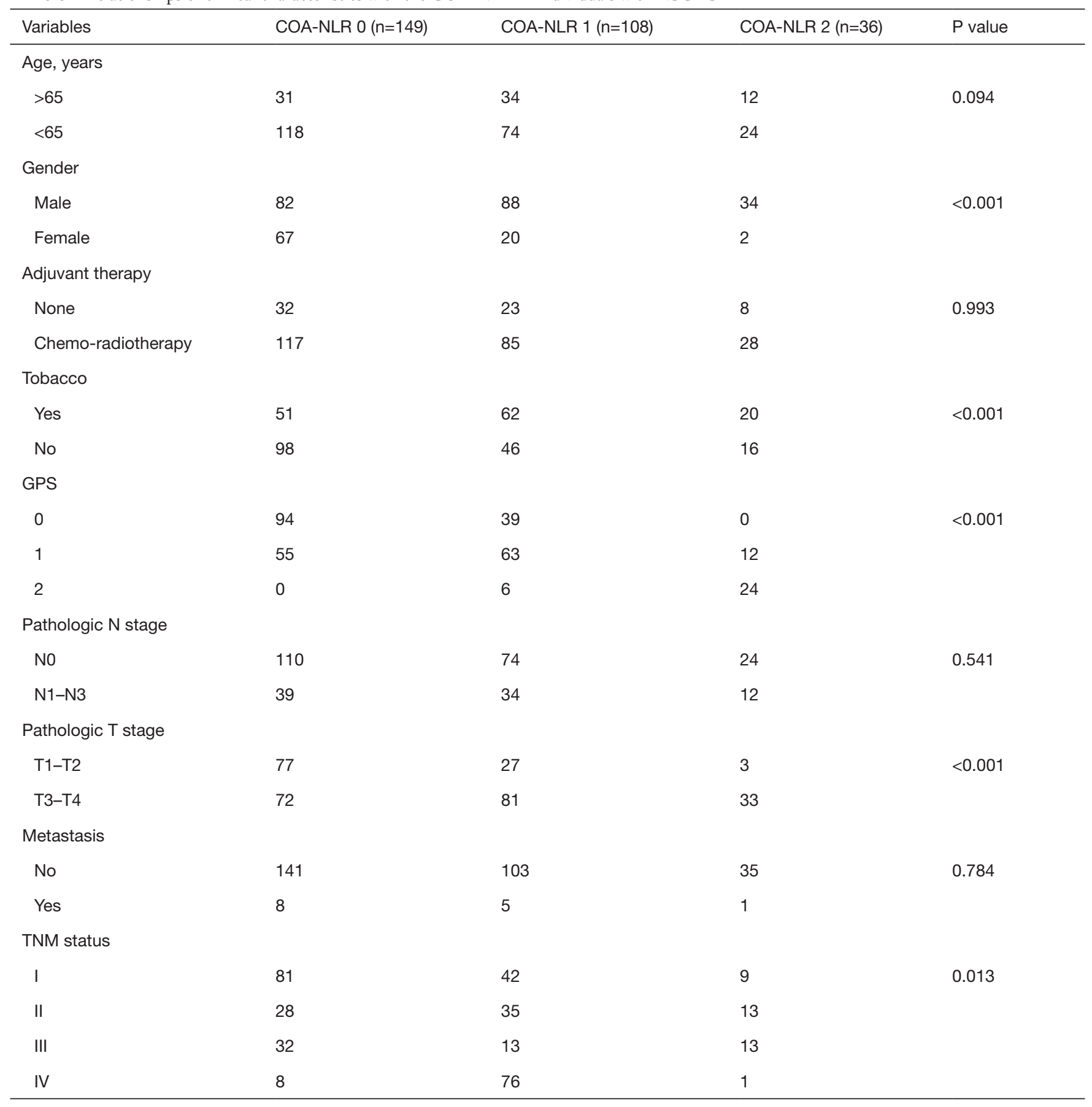

COA-NLR, combination of albumin concentration and neutrophil-to-lymphocyte ratio; NSCLC, non-small cell lung cancer; GPS, Glasgow prognostic score; TNM, tumor-node-metastasis.

progress of cancer. What's more, COA-NLR could be a sign that stands for immunity status. As inflammatory response can suppress antitumor immunity and hypoalbuminemia can impair cellular immunity. Lower score of COA-NLR means better immunity status, which was better for the outcomes of lung cancer. 
Table 2 Relationships of clinical laboratory features with the COA-NLR in individuals with NSCLC

\begin{tabular}{|c|c|c|c|c|}
\hline Variables & COA-NLR $0(n=149)$ & COA-NLR $1(n=108)$ & COA-NLR $2(n=36)$ & $P$ value \\
\hline Age, years & $57 \pm 9$ & $60 \pm 9$ & $61 \pm 10$ & 0.063 \\
\hline $\mathrm{BMI}, \mathrm{kg} / \mathrm{m}^{2}$ & $23 \pm 2.9$ & $23 \pm 3.3$ & $22 \pm 3.1$ & 0.077 \\
\hline OS (months) & $59 \pm 22$ & $49 \pm 26$ & $36 \pm 27$ & $<0.001$ \\
\hline $\mathrm{CRP}, \mathrm{mg} / \mathrm{dL}$ & $1.1 \pm 1.2$ & $1.7 \pm 1.9$ & $2.6 \pm 1.6$ & 0.001 \\
\hline WBC, $10^{9} / \mathrm{L}$ & $6.9 \pm 1.7$ & $8.5 \pm 2.2$ & $10.0 \pm 2.6$ & $<0.001$ \\
\hline Neutrophil, $10^{9} / \mathrm{L}$ & $3.8 \pm 1.2$ & $5.7 \pm 2.2$ & $7.0 \pm 2.0$ & $<0.001$ \\
\hline Lymph, $10^{9} / \mathrm{L}$ & $2.3 \pm 0.6$ & $1.8 \pm 0.5$ & $1.9 \pm 0.6$ & $<0.001$ \\
\hline NLR & $1.7 \pm 0.5$ & $3.5 \pm 2.9$ & $4.0 \pm 1.6$ & $<0.001$ \\
\hline $\mathrm{Hb}, \mathrm{g} / \mathrm{L}$ & $137 \pm 14$ & $135 \pm 15$ & $125 \pm 14$ & $<0.001$ \\
\hline PLT, $10^{\circ} / \mathrm{L}$ & $242 \pm 70$ & $257 \pm 76$ & $342 \pm 121$ & $<0.001$ \\
\hline Alb, g/L & $40 \pm 2.6$ & $37 \pm 3.9$ & $32 \pm 2.4$ & $<0.001$ \\
\hline Glucose, mmol/L & $5.5 \pm 1.0$ & $5.6 \pm 1.5$ & $5.2 \pm 1.3$ & 0.023 \\
\hline $\mathrm{LDH}, \mathrm{U} / \mathrm{L}$ & $147 \pm 24$ & $181 \pm 110$ & $139 \pm 25$ & 0.044 \\
\hline ALT, U/L & $20.9 \pm 15.9$ & $20.9 \pm 13.6$ & $29.6 \pm 30.4$ & 0.311 \\
\hline
\end{tabular}

COA-NLR, combination of albumin concentration and neutrophil-to-lymphocyte ratio; NSCLC, non-small cell lung cancer; BMI, body mass index; OS, overall survival; CRP, C-reactive protein; WBC, white blood cell; NLR, neutrophil-to-lymphocyte ratio; Hb, hemoglobin; PLT, platelet; Alb, albumin; LDH, lactate dehydrogenase; ALT, alanine transaminase.

Low Alb is common in patients with cancer, as a negative acute-phase protein, it usually integrates with other biomarkers to create novel prognostic signatures, for instance modified GPS (mGPS) (8). Research has documented that the existence of a SIR precedes the development of hypoalbuminemia in cancer patients with poor prognosis $(5,9-11)$. However, the relationship between low Alb and OS have rarely been studied in NSCLC patients. Herein, we found that reduced Alb may be linked to poor OS in individuals with NSCLC $(\mathrm{P}<0.001)$. The role of NLR in lung cancer was addressed in the previous report (12). High NLR, revealing an elevated inflammatory reaction (dependent on neutrophils) along with a suboptimal lymphocyte-triggered antitumor immune reaction, has been linked to decreased OS in NSCLC (13).

To the best of our knowledge, no studies had previously investigated the relationship of NLR with serum Alb in individuals with NSCLC. Herein, we found that patients with decreased Alb may have shorter OS. Scoring systems that combine NLR and Alb were created, and participants were stratified into three classes. This scoring system was particularly successful in estimating differences among the three study groups in OS in multivariate models. Subgroup analyses confirmed the validity of this stratification approach. Hence, NLR and serum Alb level were sensitive prognostic markers that provided additional risk stratification for individuals with NSCLC.

Compared with other prognostic biomarkers, such as GPS and TNM status, COA-NLR are more suitable for predicting the outcome in patients with NSCLC. We found that COA-NLR score classified patients into three independent groups, which can more effective to stratifies prognosis than TNM status.

Our findings showed complex cross talks of systematic inflammation with the progress of tumors. Both Alb and the NLR may play various roles in systematic inflammation, which lead to the progress of cancer. Serum Alb is mainly synthesized in the liver (14), and reduced pre-operative Alb may indicate a malnourished state (15). Meanwhile, in cancer patients, Alb could reflect sustained systemic inflammation (16-18), low Alb suggests a suppressed immune index. In the immune system, $\mathrm{T}$ lymphocytes are pivotal adaptive immune cells, commonly categorized into two subsets on the basis of the expression of CD4 and CD8 receptors (19). Reduced lymphocyte levels can result in immunosuppression, leading to production of inflammatory 
Table 3 Univariate Cox proportional hazards model analysis of OS in individuals with NSCLC

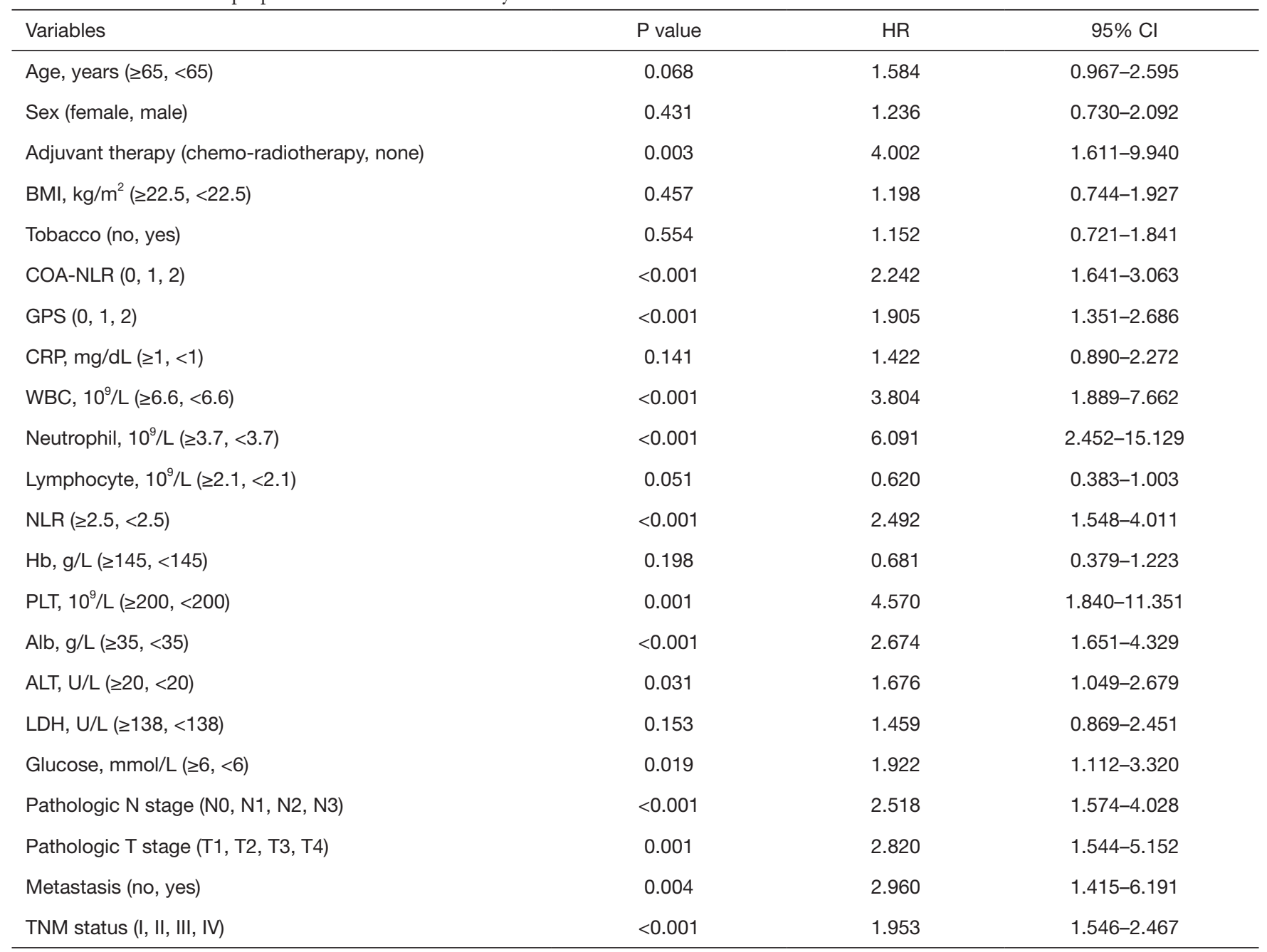

OS, overall survival; NSCLC, non-small cell lung cancer; HR, hazard ratio; CI, confidence interval; BMI, body mass index; COA-NLR, combination of albumin concentration and neutrophil-to-lymphocyte ratio; GPS, Glasgow prognostic score; CRP, C-reactive protein; WBC, white blood cell; NLR, neutrophil-to-lymphocyte ratio; Hb, hemoglobin; PLT, platelet; Alb, albumin; ALT, alanine transaminase; LDH, lactate dehydrogenase; TNM, tumor-node-metastasis.

cytokines in the tumor microenvironment (TME) (20).

Consequently, COA-NLR emerges as a good predictive indicator for individuals with NSCLC. The OS was shorter for high COA-NLR at baseline in contrast with the low COA-NLR at baseline. The COA-NLR escalates the unfavorable impact of Alb, as well as NLR, which elevates the estimated significance for individuals with cancer. Multivariate analyses conducted using the selected parameters in the univariate analyses showed that preoperative COA-NLR was an independent predictive indicator for individuals with NSCLC with OS. Moreover, data of the Kaplan-Meier test along with log-rank test illustrated that pre-operative COA-NLR scores could well stratify patients with NSCLC into different risk categories.

This study had numerous limitations. Firstly, this was a single center, retrospective analysis, that enrolled only those patients with complete volumetric measurement, which may have resulted in selection bias. Secondly, the study's sample size was small. There were only 36 participants with high score COA-NLR, a larger amount of data is required to verify these results. Thirdly, although we restricted the impact of some other factors, blood cell counts may have been affected by various factors.

The COA-NLR prospectively emerges as cost-friendly, 
Table 4 Multivariate Cox proportional hazards model analysis of OS in individuals with NSCLC

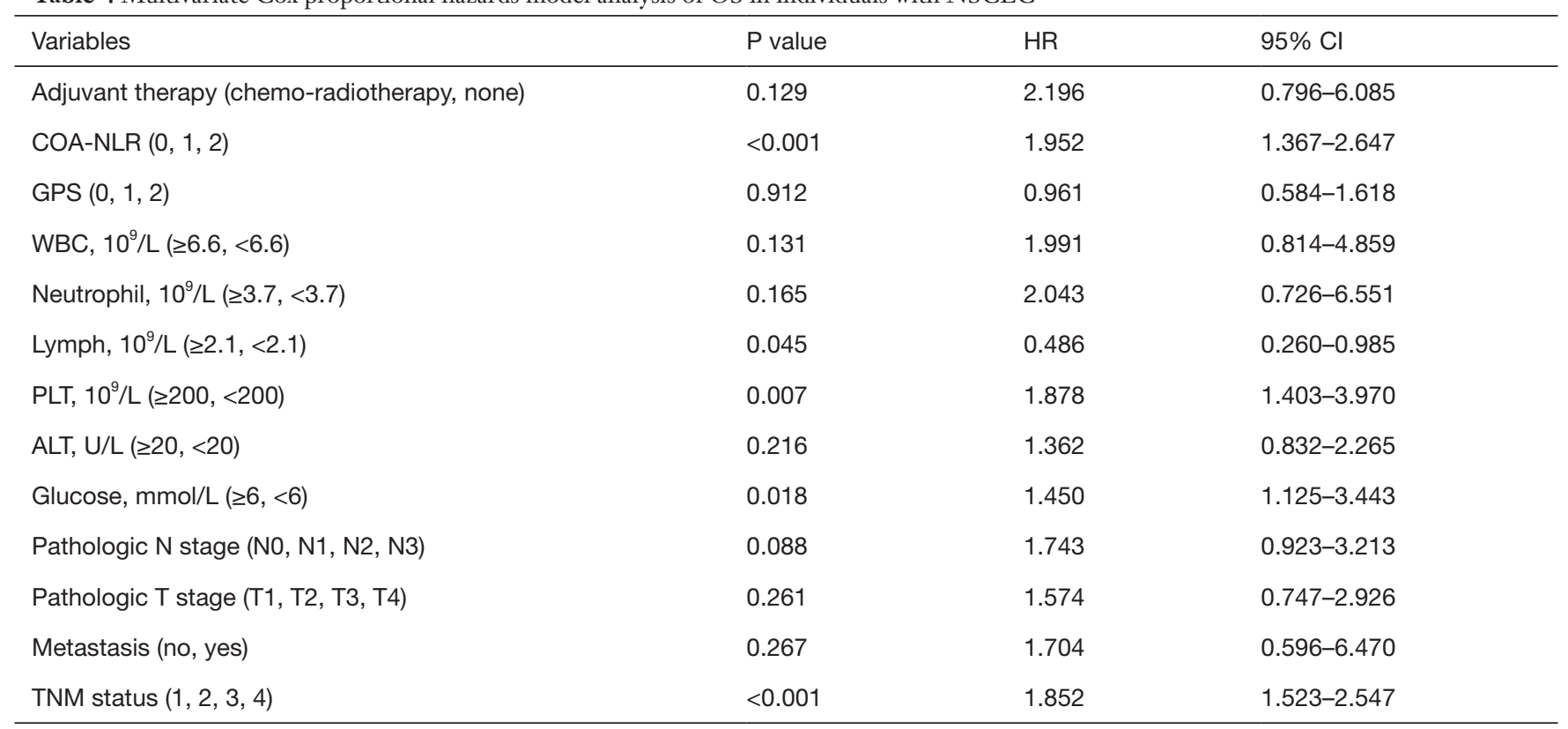

OS, overall survival; NSCLC, non-small cell lung cancer; HR, hazard ratio; Cl, confidence interval; COA-NLR, combination of albumin concentration and neutrophil-to-lymphocyte ratio; GPS, Glasgow prognostic score; WBC, white blood cell; PLT, platelet; ALT, alanine transaminase; TNM, tumor-node-metastasis.
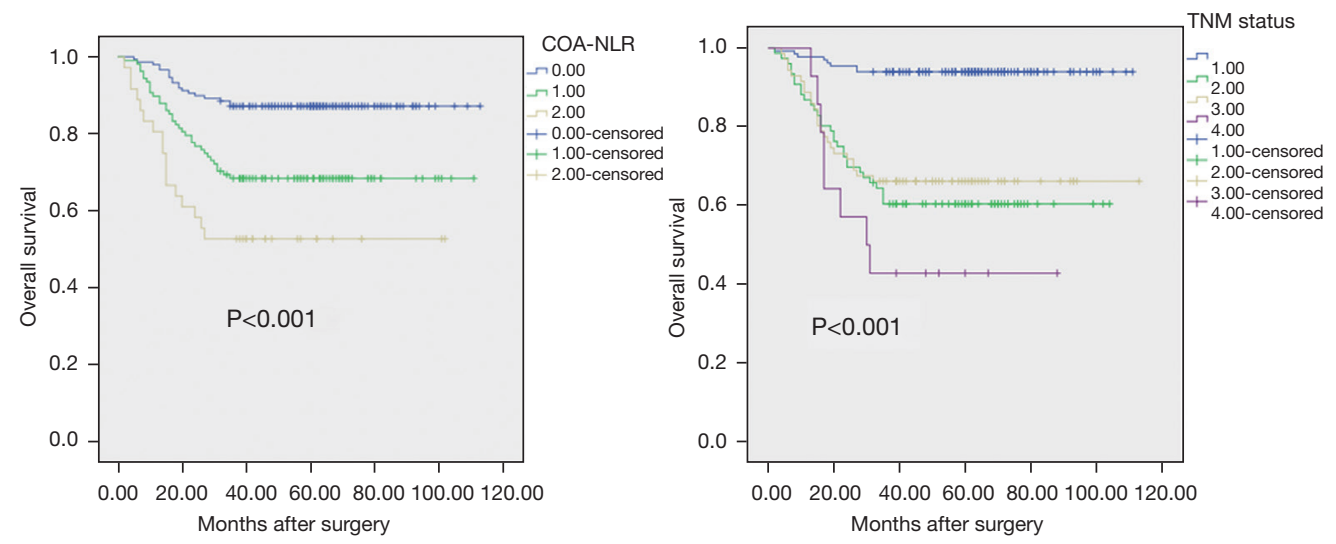

Figure 1 A schematic representation of the Kaplan-Meier graph: OS according to COA-NLR (left) and OS according to TNM status (right) in lung cancer patients undergoing surgery. OS, overall survival; COA-NLR, combination of albumin concentration and neutrophil-tolymphocyte ratio; TNM, tumor-node-metastasis.

simple, robust, as well as reliable tool accessible to all clinics and easy to adopt into clinical practice. These results could enhance treatment outcomes via informing risk stratification and individual decision-making in recently diagnosed NSCLC patients. Preoperative COA-NLR score can be a useful predictive marker for NSCLC patients with lung resection.

\section{Acknowledgments}

Funding: None.

\section{Footnote}

Reporting Checklist: The authors have completed the 
REMARK reporting checklist. Available at https://dx.doi. org/10.21037/jtd-21-1320

Data Sharing Statement: Available at https://dx.doi. org/10.21037/jtd-21-1320

Conflicts of Interest: All authors have completed the ICMJE uniform disclosure form (available at https://dx.doi. org/10.21037/jtd-21-1320). The authors have no conflicts of interest to declare.

Ethical Statement: The authors are accountable for all aspects of the work in ensuring that questions related to the accuracy or integrity of any part of the work are appropriately investigated and resolved. The study was conducted in accordance with the Delaration of Helsinki (as revised in 2013). This study was approved by the Ethics Committees of the Fujian Medical University Cancer Hospital \& Fujian Cancer Hospital, Fuzhou, China, which waived the requirement for informed consent due to the use of anonymized retrospective data that were routinely collected during the health-screening process.

Open Access Statement: This is an Open Access article distributed in accordance with the Creative Commons Attribution-NonCommercial-NoDerivs 4.0 International License (CC BY-NC-ND 4.0), which permits the noncommercial replication and distribution of the article with the strict proviso that no changes or edits are made and the original work is properly cited (including links to both the formal publication through the relevant DOI and the license). See: https://creativecommons.org/licenses/by-nc-nd/4.0/.

\section{References}

1. Vansteenkiste J, De Ruysscher D, Eberhardt WE, et al. Early and locally advanced non-small-cell lung cancer (NSCLC): ESMO Clinical Practice Guidelines for diagnosis, treatment and follow-up. Ann Oncol 2013;24 Suppl 6:vi89-98.

2. Haseltine JM, Rimner A, Shepherd AF, et al. Delivering safe and effective stereotactic body radiation therapy for patients with centrally located early stage non-small cell lung cancer. Chin Clin Oncol 2020;9:39.

3. Brcic L, Kern I. Outsourcing predictive biomarker testing in non-small cell carcinoma: a personal view of pathologists. Transl Lung Cancer Res 2020;9:2194-8.

4. Łochowski M, Łochowska B, Zawadzka I, et al. Prognostic value of neutrophil-to-lymphocyte, platelet-to-lymphocyte and lymphocyte-to-monocyte ratio ratios in patients operated on due to non-small cell lung cancer. J Thorac Dis 2019;11:3377-84.

5. Xia L, Huang H, Xiao H, et al. Utilization of combined PD-L1 expression and neutrophil-to-lymphocyte ratio prior to surgery as a prognostic factor in non-small cell lung cancer with brain metastasis. Transl Cancer Res 2019;8:2864-77.

6. Takahashi Y, Kawamura M, Hato T, et al. Neutrophillymphocyte ratio as a prognostic marker for lung adenocarcinoma after complete resection. World J Surg 2016;40:365-72.

7. Goldstraw P, Crowley J, Chansky K, et al. The IASLC Lung Cancer Staging Project: proposals for the revision of the TNM stage groupings in the forthcoming (seventh) edition of the TNM Classification of malignant tumours. J Thorac Oncol 2007;2:706-14.

8. Ni XC, Yi Y, Fu YP, et al. Prognostic value of the modified Glasgow prognostic score in patients undergoing radical surgery for hepatocellular carcinoma. Medicine (Baltimore) 2015;94:e1486.

9. Xu XL, Yu HQ, Hu W, et al. A novel inflammation-based prognostic score, the C-reactive protein/albumin ratio predicts the prognosis of patients with operable esophageal squamous cell carcinoma. PLoS One 2015;10:e0138657.

10. Yamashita K, Ushiku H, Katada N, et al. Reduced preoperative serum albumin and absence of peritoneal dissemination may be predictive factors for long-term survival with advanced gastric cancer with positive cytology test. Eur J Surg Oncol 2015;41:1324-32.

11. Zhou T, Zhan J, Hong S, et al. Ratio of C-reactive protein/ albumin is an inflammatory prognostic score for predicting overall survival of patients with small-cell lung cancer. Sci Rep 2015;5:10481.

12. Guthrie GJ, Charles KA, Roxburgh CS, et al. The systemic inflammation-based neutrophil-lymphocyte ratio: experience in patients with cancer. Crit Rev Oncol Hematol 2013;88:218-30.

13. Gu XB, Tian T, Tian XJ, et al. Prognostic significance of neutrophil-to-lymphocyte ratio in non-small cell lung cancer: a meta-analysis. Sci Rep 2015;5:12493.

14. Saraiva MJ, Magalhaes J, Ferreira N, et al. Transthyretin deposition in familial amyloidotic polyneuropathy. Curr Med Chem 2012;19:2304-11.

15. Quero Alfonso AI, Fernández Castillo R, Fernández Gallegos R, et al. Study of serum albumin and BMI as nutritional markers in hemodialysis patients. Nutr Hosp 
2014;31:1317-22.

16. Ishida S, Hashimoto I, Seike T, et al. Serum albumin levels correlate with inflammation rather than nutrition supply in burns patients: a retrospective study. J Med Invest 2014;61:361-8.

17. Dashti N, Einollahi N, Nabatchian F, et al. Significance of albumin and C-reactive protein variations in 300 end stage renal disease patients in Tehran University of Medical Sciences Hospitals during year 2010. Acta Med Iran 2012;50:197-202.

18. Powell AGMT, Eley C, Chin C, et al. Prognostic significance of serum inflammatory markers in esophageal

Cite this article as: Weng J, Huang J, Yu W, Zhao Z, Zhu B, Lin J, Cai Y, Zhang J, Su W, Chen X, Zhu K, Lin S. Combination of albumin concentration and neutrophil-tolymphocyte ratio for predicting overall survival of patients with non-small cell lung cancer. J Thorac Dis 2021;13(9):5508-5516. doi: $10.21037 /$ jtd-21-1320 cancer. Esophagus 2021;18:267-77. Erratum in: Esophagus 2021;18:710.

19. Overgaard NH, Jung JW, Steptoe RJ, et al. CD4+/CD8+ double-positive $\mathrm{T}$ cells: more than just a developmental stage? J Leukoc Biol 2015;97:31-8.

20. Kwilas AR, Donahue RN, Tsang KY, et al. Immune consequences of tyrosine kinase inhibitors that synergize with cancer immunotherapy. Cancer Cell Microenviron 2015;2:e677.

(English Language Editor: J. Jones) 